\title{
68. CONTRIBUTO ALLO STUDIO DEI FUNGHI FIMICOLI IV. MYXOMYCETES. DIDYMIUM SQUAMULOSUM (ALB. \& SCHWEIN.) Fr. E PERICHAENA CORTICALIS (BATSCH) ROSTAF.
}

\section{Gabriele CACIALLI, Vincenzo CAROTI e Francesco DOVERI}

Contribution to the study of fimicolous fungi IV. Myxomycetes. Didymium squamulosum (Alb. \& Schweim). Fr. and Perichaena corticalis (Batsch) Rostaf.

Paroles chiave. Fimicoli, funghi, Myxomycetes.

Key words.Fimiculous, fungi, Myxomycetes.

Il censimento della micoflora fimicola italiana ci ha permesso di studiare alcune specie molto rare e addirittura un taxon nuovo per la Scienza, ma nella maggior parte dei casi ci ha proposto delle entità piuttosto comuni, tra le quali dobbiamo annoverare Didymium squamulosum e Perichaena corticalis. Ad entrambi è stata ugualmente dedicata una particolare attenzione, che è culminata con la pubblicazione di questo contributo. Siamo stati colpiti dalla grande variabilità morfologica di Didymium squamulosum e, cercando di capirne le cause tramite la consultazione della letteratura specializzata, abbiamo scoperto tra l'altro la sua notevole adattabilità al substrato di crescita. Per quanto concerne invece Perichaena corticalis siamo stati attratti dalle difficoltà diagnostiche che si incontrano all'interno di quello che può essere definito "complesso" o "gruppo" corticalis.

Didymium squamulosum (Alb. \& Schwein.) Fr., Symb. Gast. 19, 1818.

Bas.: Diderma squamulosum Alb.\& Schwein., Consp. Fung. 88, 1805.

= Licea stipitata DC., Fl. Fr. 5: 101, 1815.

= Tubulina pedicellata Poir. in Lam., Encyc. Suppl. 5: 373, 1817.

= Didymium costatum Fr., Syst. Myc. 3: $118,1829$.
= Didymium herbarum Fr., Syst. Myc. 3: 120, 1829.

= Physarum liceoides Duby, Bot. Gal., $861,1830$.

= Didymium praecox de Bary in Rabenh., Fungi Eur. 367, 1861.

= Didymium bonianum Pat., Jour. Bot., 5: 316,1891 .

Materiale: Italia, Pisa, Calambrone, $0 \mathrm{~m}$, circa cinquanta fruttificazioni gregarie su escrementi di cavallo (Equus caballus), F. Doveri, 18.6.94., 272.2.-Marina di Pisa, MCVE 487.

Fruttificazioni alte 700-1000 $\mu \mathrm{m}$, sotto forma di sporocisti stipitate, subpeduncolate 0 sessili, eccezionalmente con l' aspetto di brevi plasmodiocarpi. Parte superiore larga 300-400 $\mu \mathrm{m}$, globosa o subglobosa, leggermente ombelicata alla base.

Peridio membranoso, ricoperto uniformemente da una fine crosta calcarea biancastra o grigia chiara, di aspetto farinoso, che si dissocia a maturazione, formando una rete a maglie sempre più larghe, attraverso le quali si osserva il capillizio cotonoso e le spore nerastre in massa.

Stipite calcareo, alto $370-500 \mu \mathrm{m}$, largo 100-150 $\mu \mathrm{m}$, allargato alla base, rastremato all' apice, bianco o arancione, appena scanalato in senso longitudinale, leggermente 
tubercoloso (caratteristica osservabile soltanto con lente a forte ingrandimento).

Columella presente, discoidale, calcarea. Ipotallo più o meno concolore allo stipite.

Spore 9,5-10 x 9-10 $\mu \mathrm{m}$, globose o subglobose $(\mathrm{Q}=1,0-1,05)$, marroni-violette in acqua, con pareti spesse, ornamentate con spinule molto fitte, isolate, raramente confluenti alla base, poco pronunciate (alte e larghe 0,2-0,3 $\mu \mathrm{m}$ ).

Capillizio ialino, semplice o ramificato, composto da filamenti larghi 1-2 $\mu \mathrm{m}$, che in alcuni punti formano dei nodi fino a $5 \mu \mathrm{m} \mathrm{di}$ diametro; pareti sottili, forforose per la presenza di incrostazioni molto fini.

Calcare: cristallino a livello del peridio, granuloso sullo stipite.

Osservazioni. Didymium squamulosum si presenta costantemente sotto forma di sporocisti stipitate, ma paradossalmente la facilità con la quale questa specie può essere inquadrata all'interno del genere di appartenenza dipende dalla variabilità morfologica dei corpi fruttiferi. In una medesima collezione possono essere osservate infatti, accanto alle forme tipiche, delle sporocisti substipitate o sessili, accompagnate talvolta da qualche breve plasmodiocarpo. Anche lo stipite calcareo, alto, robusto e scanalato ed il peridio, con componente calcarea intimamente associata a quella membranosa, contribuiscono alla corretta definizione di questo taxon.

La nostra specie cresce indifferentemente sia su sostanze vegetali (piante secche, lettiere di foglie, legno in decomposizione) che su escrementi di erbivori, spontaneamente $o$ in coltura in camera umida. Mentre alcuni Myxomycetes fimicoli sono in grado di svilupparsi esclusivamente su sterco di animali domestici ed altri (ad es. Stemonitis fusca Roth) vengono ottenuti in coltura solo su escrementi di animali selvatici (Eliasson \& Lundqvist, 1979), Didymium squamulosum non dimostra alcuna preferenza né per gli uni né per gli altri e, come quasi tutti i Myxomycetes che hanno la facoltà di crescere su escrementi, non si comporta da endocoprofilo puro ma semplicemente da fimicolo secondario. Lo sterco degli animali erbivori fornisce un ottimo terreno di crescita per molti Myxomycetes, in quanto è in grado di mantenere una buona percentuale di umidità e di trattenere il calore derivante dall'irradiazione solare anche quando la temperatura esterna è molto bassa (Gøtzsche, 1989).

PERICHAENA CORTICALIS (Batsch) Rostaf., Mon. 293, 1875

Bas.: Lycoperdon corticale Batsch, Elench. Fung. 155, 1783.

= Sphaerocarpus sessilis Bull., Hist. Champ. Fr. 132, 1791.

= Trichia gymnosperma Pers., Obs. Myc. 1: 63, 1796.

= Licea circumscissa (Schrad.) Pers., Syn. Fung., 196, 1801.

= Physarum luteoalbum Schumach., Enum. Pl. Saell. 2: 199, 1803.

= Perichaena abietina Fr., Symb. Gast. 11, 1817.

= Perichaena populina Fr., Symb. Gast.

12, 1817.

= Pyxidium sessile S. F. Gray, Nat. Arr. Brit. Plant. 1: 580, 1821.

= Perichaena rostafinskyi P. Karst., Bidr. Finl. Nat. Folk 31: 130, 1879.

Materiale: Italia, Livorno, Botro delle Fontanelle, $200 \mathrm{~m}$, una decina di fruttificazioni su escrementi bovini (Bos taurus) in coltura, $\mathrm{F}$. Doveri, 1) 15.5.95., 284.4.-Collesalvetti, MCVE 488. 2) 16.6.95., 284.4.-Collesalvetti, CLSM 01095 bis.

Fruttificazioni sotto forma di sporocisti gregarie, talvolta appressate ma non ammucchiate, sessili, globose, arancioni- 
rossastre scure, piuttosto brillanti, lisce, membranose, $210-400 \mu \mathrm{m}$ di diametro.

Spore 12,5-14 x 11-13 $\mathrm{m}$, da subglobose a largamente ellissoidali, gialle dorate brillanti, a pareti spesse, rivestite da fitte verruche isolate, molto basse, talvolta puntiformi.

Capillizio largo 1,5-4,5 $\mu \mathrm{m}$, tubulare, giallo dorato o ialino a livello delle ramificazioni più sottili, costituito sia da brevi elateri ad estremità libera arrotondata (raramente appuntita) che da lunghi rami anastomizzati, formanti un reticolo a maglie incomplete. I tubuli più larghi presentano numerosi rigonfiamenti nodulari, fino a $9 \mu \mathrm{m}$ di diametro, mentre sull'intero capillizio si notano alcuni diverticoli, saltuari restringimenti della parete a forma di anello ed una verrucosità molto fine, sparsa, osservabile con grande difficoltà anche a forte ingrandimento.

Peridio a due strati: l'esterno granulare, l'interno membranoso, laminare.

N.B.: Il capillizio della collezione "01095 bis" presenta una verrucosità fine, più fitta e più evidente di quella osservata in precedenza, e delle spine ben delineate. Le spore sono leggermente più grandi, con diametro massimo di $15 \mu \mathrm{m}$. Da notare che in alcuni esemplari, a causa forse dell'insufficiente illuminazione, le spore ed il capillizio sono particolarmente chiari, quasi ialini.

Osservazioni. P.corticalis, insieme a Perichaena depressa ed a Perichaena chrysosperma (Curr.) Lister, fa parte di un complesso di specie che si adatta molto bene a diversi substrati di crescita; infatti queste entità si sviluppano normalmente sulla corteccia di rami morti, ma in coltura possono essere ottenute con estrema facilità anche su escrementi di erbivori (Martin \& Alexopoulos, 1969; Eliasson \& Lundqvist, l.c.; Cochet, 1977)

P.depressa si contraddistingue per la regolarità della fessura di deiscenza peridiale (ondulata in P.corticalis) e per la compressione reciproca delle sferocisti, che di conseguenza risultano poligonali ed appiattite (isolate e globose quelle di P.corticalis); le spore sono più piccole, anche se non im maniera significativa, di quelle della specie da noi descritta.

In Perichaena chrysosperma è molto irregolare la deiscenza delle fruttificazioni, che si presentano quasi sempre sotto forma di brevi plasmodiocarpi anulari, talvolta associati a sporocisti; le spore sono più piccole, il capillizio spinoso.

Dobbiamo tuttavia precisare che le differenze tra queste entità non sempre sono così nette: molta luce deve essere ancora fatta, secondo il nostro punto di vista, sui rapporti che legano P.corticalis a P.depressa ed a P.chrysosperma, soprattutto se consideriamo che in coltura possono svilupparsi degli intermedi, che rendono estremamente complessa la diagnosi a livello specifico (Martin \& Alexopoulos, l.c.). Nasce di conseguenza il sospetto di trovarci molto spesso di fronte a forme o a varietà di un medesimo taxon, come osservato ad esempio da Eliasson \& Lundqvist (l.c.). Anche Perichaena depressa è estremamente variabile: il capillizio di questa specie viene descritto da Martin \& Alexopoulos (l.c.), ma non da altri studiosi, come abbondante $o$, al contrario, molto ridotto, ricco di espansioni nodulari (presenti anche nei nostri campioni), spinoso o minutamente verrucoso o provvisto di costrizioni anulari; le sporocisti, quando non sono troppo appressate, assumono un aspetto pulvinato.

In conclusione definiamo la nostra specie Perichaena corticalis, ma ci proponiamo di approfondire lo studio di questo "complesso", considerando anche la facilità con la quale le specie ad esso appartenenti possono essere ottenute in coltura. Le caratteristiche strutturali delle entità che si sviluppano in camera umida sono talvolta un po' diverse da quelle delle specie omologhe, cresciute allo stato naturale. Si ripropone ancora una volta l'annoso problema della variabilità infraspecifica, i cui limiti 
talvolta non sono ben definibili, con il risultato che troppo spesso dobbiamo assistere alla creazione di taxa superflui.

\section{BIBLIOGRAFIA}

COCHET, S., -1977- Les Myxomycètes de France. Bull. Soc. Myc. Fr. 93 (3): 159-200.

ELIASSON, U. \& N. LUNDQVIST -1979- Fimicolous Myxomycetes. Bot. Not. 132: 551-568.

GØTZSCHE, H.F. -1989- Myxomycetes from Greenland. Opera Botanica 100: 93-103.
MARTIN, G.W. \& C.J. ALEXOPOULOS -1969The Myxomycetes. Univ. Iowa Press.

Aceptado para su publicación en Enero de 1998

Dirección de los autores. G. Cacialli: via Aloisi 3, 57128 Livorno (Italia). V. Caroti: via Zola 51, 57122 Livorno (Italia). F. Doveri: via Baciocchi 9, 57126 Livorno (Italia).

\section{GIGASPERMUM MOURETII CORB. (GIGASPERMACEAE, MUSCI), A NEW SPECIES FROM ITALY}

\section{Alfredo CARRATELLO \& Michele ALEFFI}

Gigaspermum mouretii Corb. (Gigaspermaceae, Musci), una nueva especie para Italia

Key words. Musci, Gigaspermum, Italy.

Palabras clave. Musci, Gigaspermum, Italia.

During a series of studies conducted in north-western Sicily in October 1996, Gigaspermum mouretii Corb. was gathered by A. Carratello in the periphery of the inhabited center of the municipality of Capaci (Palermo) (3810'93" N, 1314'28”E). The species was found on a small piece of land (about $200 \mathrm{~m}^{2}$ ) once used for pasture, at about $50 \mathrm{~m}$ from the sea and at $5 \mathrm{~m}$ a.s.l., with $\mathrm{N}-\mathrm{NW}$ exposure.

Its leaves are evenly spaced or crowded, almost orbicular, apiculate, concave and 0.7 $\mathrm{mm}$ long (without the apiculus) (fig. 1). Its margin is flat, smooth and somewhat crenulate in the upper part. Nerves are absent. Cells in the basal part of the leaf are rectangular with smooth walls; in the median and upper part they are often partly hyaline, irregular to rhombic. The perichaetial leaves are hyaline, green at the base, extending $2.5 \mathrm{~mm}$ long; they are elongate-triangular, longly acuminate and strongly concave, enclosing the whole capsule. The margin is flat, smooth in the lower half and slightly denticulate in the upper half. Its capsule is sessile, gymnostomous, with a wide, slightly 